\title{
Mother to Child Transmission of HIV after Option B+ in Low Income Environment
}

\author{
Mve Koh Valère ${ }^{1,2^{*}}$, Kamgaing Nelly ${ }^{1,2,3}$, Nda Mefo ${ }^{4,5}$, Foumane Pascal ${ }^{1,6}$ \\ ${ }^{1}$ Faculty of Medicine and Biomedical Sciences of University of Yaoundé I, Yaoundé, Cameroon \\ ${ }^{2}$ University Teaching Hospital of Yaoundé, Yaoundé, Cameroon \\ ${ }^{3}$ Chantal Biya International Reference Center for Research on Prevention and Management of HIV, Yaounde, Cameroon \\ ${ }^{4}$ Faculty of Medicine and Pharmaceutical Sciences of the University of Douala, Douala, Cameroon \\ ${ }^{5}$ General Hospital of Douala, Douala, Cameroon \\ ${ }^{6}$ Gynecological Obstetrics and Pediatric Hospital Yaoundé, Yaoundé, Cameroon \\ Email: *vmvekoh@yahoo.com
}

How to cite this paper: Valère, M.K. Nelly, K., Mefo, N. and Pascal, F. (2018) Mother to Child Transmission of HIV after Option B+ in Low Income Environment. Open Journal of Obstetrics and Gynecology, 8, 1163-1175.

https://doi.org/10.4236/ojog.2018.812118

Received: August 27, 2018

Accepted: October 15, 2018

Published: October 18, 2018

Copyright $\odot 2018$ by authors and Scientific Research Publishing Inc. This work is licensed under the Creative Commons Attribution International License (CC BY 4.0).

http://creativecommons.org/licenses/by/4.0/

(C) (i) Open Access

\begin{abstract}
Background: In Cameroon, the prevalence of HIV in pregnant women was $7.8 \%$ in 2012, and they were $8500 \mathrm{HIV}$ positive newborns in 2013. Option B+ is the first highly active antiretroviral therapy (HAART) preventive protocol. The objective was to evaluate the rate of HIV transmission on children born from mothers who were on Option B+ during pregnancy, in three university teaching hospitals of the University of Yaoundé I. Methods: It was a retrospective, cross-sectional study over a period of four years (2013-2017). We included HIV positive mothers not on previous antiretroviral treatment and who received a single tablet daily of combined tenofovir (300 mg) + Lamivudine $(300 \mathrm{mg})+$ Efavirenz $(600 \mathrm{mg})$ started at any time during pregnancy. Newborn received nevirapine syrup according to WHO option B+ protocol. Results: 179 women were included. The average age was $33.5 \pm 2.92$ years, all ages were represented. Blood donation was the most frequent HIV positive screening opportunity, voluntary testing rate was $29 \%$ (29/179), and adherence rate was $98.9 \%$. Few male partners were involved (58/179). Premature deliveries and low birth weight were rare $(5 / 179 ; 10 / 179))$, the indication of mode of delivery was strictly obstetrical, newborn feeding choice didn't affect the transmission outcome, and the mother to child transmission rate was 2.2\% (4/179). Conclusion: Option B+ could achieve the lowest mother to child transmission ever in Cameroon and should be generalized in high endemicity low resources settings.
\end{abstract}

\section{Keywords}

Option B+, University of Yaoundé I, Cross Sectional 


\section{Introduction}

Mother to child transmission of HIV represents $90 \%$ of HIV transmission among children less than 14 years and, globally, it was still the seventh leading cause of death among children aged 10 - 14 years in 2015, and the ninth of death among adolescents (aged $10-19$ years). AIDS is indeed the leading cause of adolescent death around the world with 328 deaths every day [1].

This transmission can occur during pregnancy, labor, delivery or breastfeeding and can be eliminated if HIV positive (HIV+) mothers have access to prevention of mother to child transmission (PMTCT) programs [2]. In Cameroon, a Sub-Saharan African country at the gulf of Guinea with a population of 23 million, the prevalence of HIV in pregnant women in 2012 was 7.8\% [3], 27,000 pregnant women needed antiretroviral therapy (ART) and the overall estimation of HIV mother to child transmission rate in Cameroon was 22.1\% [4]. They were $8500 \mathrm{HIV}$ positive newborns in 2013 [5], and nearly half will die within two years if no antiretroviral treatment is initiated [6]. To tackle this situation, Cameroon, a country with generalized epidemiology, has adopted the protocol named Option $\mathrm{B}+$, to further reduce mother to child transmission of HIV since 2013. It is the administration of highly active antiretroviral therapy (HAART) to all HIV + positive pregnant women once diagnosed, regardless of CD4 count and viral load for life, and nevirapine syrup to the new born. This protocol has reduced the mother to child transmission rate from $18 \%$ to $2.2 \%$ in Ethiopia [7], and to $3.5 \%$ in Malawi [8], but no study assessing the mother to child transmission rate of HIV after option $\mathrm{B}+$ has ever been conducted in the three major university PMTCT centers in the capital city of that country, including data concerning male partners involvement and HIV-exposed-children feeding mode assessment and HIV status outcome.

The objective of our study was to evaluate the rate of HIV transmission on exposed children whose mother took Tenofovir-Lamivudine-Efavirenz once a day during pregnancy, in three university teaching hospitals of the University in the capital of the republic of Cameroon in Central Africa.

\section{Methods and Materials}

It was a retrospective, cross-sectional study over a four years period (2013-2017), conducted from January $1^{\text {st }}$ to $30^{\text {th }}$ of April 2017 in three University major PMTCT implementing and referral centers of the University of Yaoundé I, namely, the University Teaching Hospital, the Central Hospital and the Gynecological Obstetrics and Pediatric Hospital, all in Yaoundé, the capital city of the republic of Cameroon in Central Africa. Altogether, they realize more than 8000 deliveries per year including a few hundred HIV positive pregnant women a year.

We included HIV+ mothers who were not on previous ART and who received a single tablet daily of combined tenofovir $(300 \mathrm{mg})+$ Lamivudine $(300 \mathrm{mg})+$ Efavirenz $(600 \mathrm{mg})$ started at any time during pregnancy and taken at least for 
four completed weeks on the day of delivery. Newborn received nevirapine syrup for 6 weeks, according to WHO (World Health Organization) option B+ protocol. Non-consenting mothers, lost or incomplete records with missed HAART duration, mode of delivery, child PCR (polymerase chain reaction) early diagnosis result and serology status were excluded. Early detection of HIV infection in exposed children was done by dried blood spot (DBS) DNA PCR of HIV 1, performed at Chantal Biya International Reference Center for Research on Prevention and Management of HIV in Yaoundé, an important HIV research center in Sub-Saharan Africa. Exposed children's serology was performed by enzyme-linked immunosorbent essay (ELISA) test, performed in the three University affiliated Hospitals of our study. The final diagnosis was made at 18 months of age, if serology was still positive after the first done at 10 months of age.

The data were collected using a pre-tested data sheet after due maternal consent. They concerned, the socio-demographic characteristics, the status of children born from HIV positive mothers. There was no viral load or CD4 assessment before delivery according to WHO's Option B+ recommendations.

Retention was defined as $100 \%$ on time monthly ART intake, confirmed by computerized registers. This study received the ethical clearance of the ethical committee of the University of Yaoundé I. Data were analyzed using CS Pro version 6.3 and Microsoft Office Excel 2013. Statistical analysis was processed using $\mathrm{X} 2$, and $\mathrm{P} \leq 0.05$ was the statistical significance threshold.

\section{Results}

We included 179 mother and child couples. They had an average age of $33.5 \pm$ 2.92 years. The youngest and the oldest age were 15 and 43 respectively, all the ages were represented, $93 \%$ had at least middle school education level (Table 1).

\section{1) Maternal HIV data}

Blood donation and antenatal care were the main circumstances of discovery of HIV+ status (71.5\%; 128/179), retention rate was very high (99\%; 177/179), $68 \%(121 / 179)$ of current sexual partner HIV status was unknown (Table 2).

\section{2) Maternal pregnancy follows up data}

Ninety-seven per cent point two percent (174/179) attended at least 4 antenatal care (ANC), premature delivery rate was 2.8\% (5/179), 77.7\% (139/179) of children were born vaginally, $21.2 \%$ delivered by emergency caesarean section not indicated for viral load which was never taken into consideration at the moment of delivery. None of these parameters was related to child HIV status $(\mathrm{P}>0.05)$ (Table 3).

Artificial milk and breast milk feeding were equally adopted in post-natal feeding option and were not related to new born HIV status (Table 4).

Post-natal HIV-exposed children HIV status assessment moment.

By 3 months post natally, $89 \%$ of HIV-exposed children had done their DBS DNA PCR screening, all the results were confirmed by serology (ELISA) at 18 months of age (Figure 1). 
Table 1. Maternal socio-demographic parameters.

\begin{tabular}{ccc}
\hline Variables & (n) & Frequency (\%) \\
\hline Ages of HIV+ mothers (in years) & & \\
{$[15-19[$} & 12 & 6,7 \\
{$[19-24[$} & 18 & 10 \\
{$[24-29[$} & 29 & 16.2 \\
{$[29-34[$} & 76 & 42.5 \\
{$[34-39[$} & 38 & 21.2 \\
$\geq 39$ & 6 & 3.3 \\
Highest educational achievement level & & \\
Never attended school & 11 & 6.2 \\
Primary school & 2 & 1.1 \\
Secondary education & 88 & 49.2 \\
University education & 78 & 43.6 \\
Marital status & & \\
Married & 82 & 45.8 \\
Single & 70 & 39.1 \\
Cohabitation with partner & 22 & 12.3 \\
Divorced & 3 & 1.7 \\
Widow & 2 & 1.1 \\
\hline
\end{tabular}

Table 2. Maternal HIV related parameters.

\begin{tabular}{ccc}
\hline Variables & $\mathbf{n}$ & Frequency (\%) \\
\hline Circumstances of HIV+ discovery & & \\
During blood donation & 87 & 48.6 \\
During current pregnancy & 41 & 22.9 \\
Voluntary testing & 29 & 16.2 \\
Testing during disease management & 22 & 12.3 \\
Duration of known HIV+ status & & \\
<4 years & 169 & 94.4 \\
$\geq 4$ years & 10 & 5.6 \\
Retention/adherence & & 98.9 \\
Yes & 177 & 1.1 \\
No & 2 & \\
Viral load unknown & & 0 \\
Yes & 0 & 100 \\
No & 179 & \\
Unknown & & 67.6 \\
Positive & 121 & 24 \\
Negative & 43 & 8.4 \\
Yurrent sexual partner HIV status & 15 & \\
No & 40 & 93 \\
HAART to HIV+ partner & 3 & 7 \\
\hline
\end{tabular}

The overall mother to child transmission rate of HIV was 2.2\% (Figure 2).

\section{Discussion}

This study as multicentric studies had limitations due to the different medical environment of the concerning PMTCT centers, the quality of male partners data due to confidentiality and those concerning HIV-exposed children feeding mode collected through mother's declaration, with corresponding bias. 
Table 3. Evaluation of obstetrical characteristics and exposed child outcome.

\begin{tabular}{ccc}
\hline Variables & $\mathbf{n}$ & Frequency (\%) \\
\hline ANC $^{*}$ & & \\
$<4$ & 5 & 2.8 \\
4 to 8 & 48 & 26.8 \\
$>8$ & 126 & 70.4 \\
Gestational age on delivery (W) & & \\
$<37$ & 5 & 2.8 \\
{$[37-40[$} & 122 & 68.2 \\
{$[40-42[$} & 52 & 29 \\
$>42$ & 0 & 0 \\
Mode of delivery* & & 77.7 \\
Vaginal delivery & 139 & 21.2 \\
Emergency caesarean section & 38 & 1.1 \\
Instrumental delivery & 2 & \\
Birth weight (g) & & 5.6 \\
$<2500$ & 10 & 94.4 \\
$\geq 2500$ & 169 &
\end{tabular}

${ }^{*} \mathrm{P}>0.05$; ANC: ante natal care; $\mathrm{W}$ : weeks.

Table 4. Post-natal child feeding mode and duration parameters.

\begin{tabular}{|c|c|c|c|c|c|c|}
\hline & & \multicolumn{5}{|c|}{ Post-natal feeding** } \\
\hline & & \multicolumn{2}{|c|}{$<6$ months } & \multicolumn{2}{|c|}{$\geq 6$ months } & \multirow[t]{2}{*}{ Total } \\
\hline & & $\mathrm{n}$ & $\%$ & $\mathrm{n}$ & $\%$ & \\
\hline \multirow{4}{*}{ 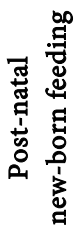 } & Artificial Milk feeding & 39 & $51.31 \%$ & 98 & $95.10 \%$ & 137 \\
\hline & Maternal feeding & 34 & $44.74 \%$ & 5 & $4.90 \%$ & 39 \\
\hline & Mixed feeding & 3 & $3.95 \%$ & 0 & $3.90 \%$ & 3 \\
\hline & Total & 76 & $100.00 \%$ & 103 & $100.00 \%$ & 179 \\
\hline
\end{tabular}

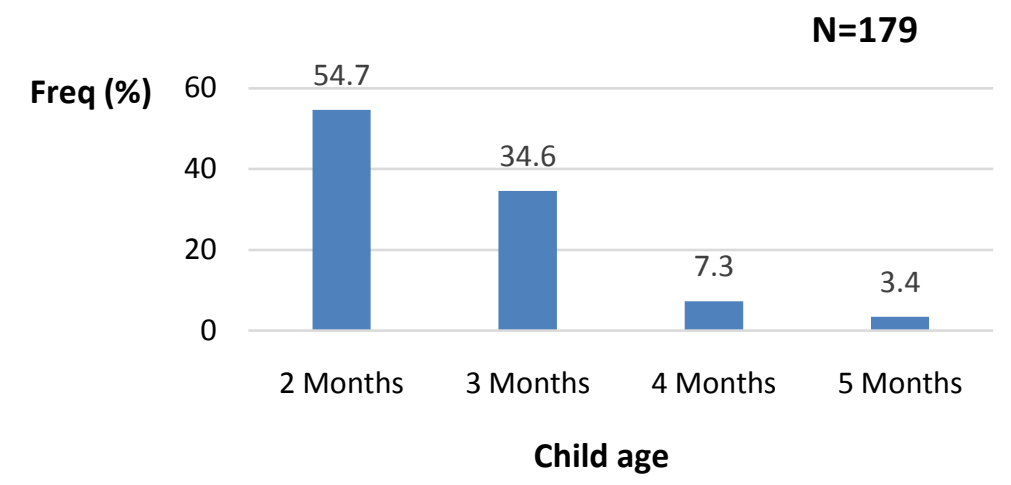

Figure 1. HIV exposed-children's age at the moment of HIV status assessment by DBS DNA PCR.

Mother to child transmission of HIV has been a public health focus in high prevalence countries like Cameroon for almost two decades. Since the HIVNET 012, first randomized trial assessing the prevention of mother to child transmission of HIV in 1999 in Uganda [9], many protocols of prevention have been 


\section{$\mathbf{N}=179$}

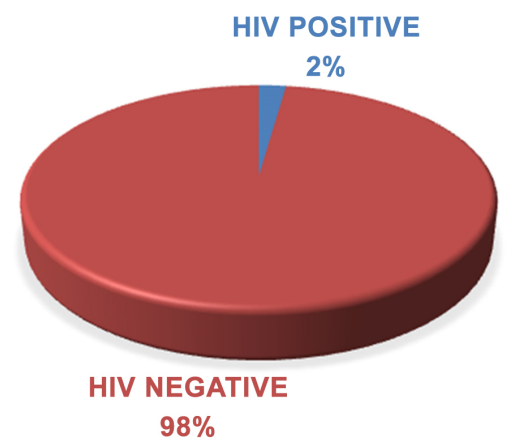

Figure 2. Rate of mother to child transmission of HIV*.

tried, looking for a better reduction of the vertical transmission rate. Option $\mathrm{B}+$ is the latest recommendation concerning the PMTCT, issued by WHO [10]. It is the first to put in place the use of HAART on pregnant women regardless of CD4 and viral load value, clinical stage or gestational age, taken during life. The recommended antiretroviral drugs used in our study were Tenofovir, lamivudine and efavirenz, given once daily, in a test and start approach. This recommendation is yet to be adopted in the western world. United Kingdom guidelines for example still relies on clinical stage, recommending ART initiation by 24 weeks of pregnancy if no indication for HAART [11].

Pregnant women of all ages were represented in our sample, showing the magnitude of the epidemy in this country. Women aged 29 - 34 years represented almost half of the study population. Those aged 15 - 24 counted for $16.7 \%$ (20/179). The frequency of HIV + pregnant women aged 15 - 24 years in Cameroon is unknown. That age group is more at risk than the others. In sub-Saharan Africa, women aged 15 - 24 made up 66\% of new infections among young people, the highest in the world, the proportion of women aged 15 - 24 years in new infections varying from $29 \%$ in Western and Central Europe and North America to $48 \%$ in the Middle East and North Africa [12]. That low frequency was probably due to the fact that new cases, meaning those discovered during pregnancy only represented $22.9 \%$ (41/17), and, the cut-off point of our duration of known HIV positive status was more or less four years. A shorter period assessment could have better shown the burden of HIV among pregnant women aged 15 - 24. Moreover, low rates of HIV diagnosis and treatment initiation among young people aged 15 - 24 still remains a challenge in the world [13].

The paradigm of antiretroviral prevention of mother to child transmission of HIV has changed, from short course antiretroviral preventive therapies to lifelong HAART treatment, thanks to the lessons learnt from the publication of Cohen et al., showing the advantages of early treatment of HIV regardless of CD4 count [14]. Lifelong HAART is more efficient in reducing viral load and increasing CD4 count than option A monotherapy, reducing the risk of com- 
munity transmission, rending possible the end of the epidemy enshrined in the new strategy called the "three 90".

Circumstances of discovery of HIV status in our study revealed the difficulties of voluntary self-screening behavior in Africa, although living in a high prevalence Sub-Saharan Africa, regardless of national and multisectoral strategies in the fight against HIV, with voluntary HIV free of charge screening campaign. Less than one fifth did it through voluntary testing indeed, whereas the 2014 Cameroon Multiple Indicators Cluster Survey (MICS) has shown that $83.4 \%$ of women aged 15 - 49 years knew how and where to do HIV test [15]. The HIV positive status had been known for less than four years for most of them. One of the strategies of the fight against HIV in Cameroon is to improve the accessibility of HIV care, with free of charge HIV screening for pregnant women, drastic reduction of $\mathrm{CD} 4$ count and Viral load coast, free of charge ART in a country without national health care insurance.

The fear of HIV positive result, stigma, misconception of HIV testing and the fear of negative consequences are among major obstacles for using the testing services [16] [17] [18], sometimes leading to cases of refusal to provide care to people with HIV reported in the western world [19]. Women living with HIV are even more likely to experience violence [20]. A systematic review revealed that higher education is a common facilitator of HIV testing and, patient's decision-making towards HIV screening can be influenced by marital dynamics, partner relationship and relationship with the health care services [18]. Those findings though not investigated in our study, probably also apply in our study population.

This study has also shown the enrolment power of antenatal care, in a context of low HIV self-screening behavior. It also permitted the initiation of lifelong HAART treatment on all the 179 women for the very first time. One of the methods to promote HIV testing among pregnant women is the "opt out" approach recommended by the CDC since 2006 [21]. It is a compulsory daily practice today in Cameroon. Pregnant women are just told that an HIV test will be included in the standard group of prenatal tests and, unless they decline, they will receive an HIV test. That approach is less time consuming than the "opt in", the classical pretest counselling, and was the screening method adopted in this study. A recent review has indeed shown that "opt out" was as good as "opt in" in detecting new cases of HIV infection [22]. Blood donation represented the main screening occasion probably due to the fact that in Cameroon, two donors are requested by blood bank institutes before selling each pine of blood for blood transfusion, becoming an important HIV screening opportunity.

One of the main problems of the new strategy is the adherence, which is the proportion of patients taking ART without missing any single day of prescription over the whole pregnancy period. With this test and treat attitude, more and more HIV+ patients are bound to take a lifelong compelling treatment, without symptoms which, if there, might have strengthen the will of abiding by drug 
prescription, in order to avoid falling seek again. Our adherence rate was surprisingly very high (99\%). A study conducted in 2015 in the University Teaching Hospital, a PMTCT center also involved in this study, identified the bad perception of HIV infection, poverty, stigmatization, low involvement of the partners, and misunderstanding of the PMTCT as factors reducing the adherence rate, but it was a patient recorded data and they randomly included 61 women attending ANC without any consideration of their real ART adherence status in a six months period [23], whereas our adherence assessment was based on computerized software registers. Another study of pregnant women living with HIV from four African countries found that women who were diagnosed with HIV before their pregnancy as were $77 \%$ of our sample were more likely to adhere to PMTCT treatment than women who tested positive during pregnancy [24]. In a qualitative study, Gugsa et al. identified Improved counseling at initiation, active follow-up counseling, women's economic empowerment interventions, promotion of peer counseling schemes as measures to improve adherence [25]. The hypothesis of the contribution of the strong desire of a HIV free newborn not to repeat the stigma raised above for the coming child, the free of charge antiretroviral drug taken once daily, the relatively short duration of pregnancy window observance assessment as compared to lifelong observance assessment and the therapeutic education aimed at strengthening observance as factors explaining this adherence not assessed in this study are to be confirmed

The involvement of male partner is important for HIV test uptake and case finding [26]. For Flax et al., testing pregnant women for HIV without their husbands, can lead to either hiding their status or disclosing, which may threaten their relationships with spouses and families [27], but cultural barriers can prevent the active involvement of male partners [28], moreover, according to the first 90 of the "three 90" strategy which aims at enrolling at least $90 \%$ of HIV positive in a given community, the male sexual partner is the main target among the so called index testing for the elimination of the HIV epidemy [26]. Unfortunately, as shown in our study, the male partner involvement was yet to be optimal. More than half of pregnant women were not even aware of their sexual partner's HIV status, and so was probably the awareness of the male partner about HIV positive status of these women for the reasons raised before [29], raising the difficulties ahead for the full implementation of the "three 90" in Africa. All the HIV positive male partners were on ART probably because Cameroon has indeed adopted the "test and treat" strategy.

The majority of our study population delivered vaginally, there was no single case of elective caesarian section indicated for HIV status, nor need of viral load considerations, another advantage in resource-limited settings like Cameroon. Elective cesarean section has been proven to reduce the rate of mother to child transmission of HIV [30] and is still recommended for women with $>400 \mathrm{HIV}$ RNA copies/ml at the time of delivery in the western world [11], but this is not recommended anymore for the PMTCT in Cameroon. In a systematic review 
indeed, Kennedy et al. have shown that the preventive effect of elective caesarian section was not statistically significant in the context of HAART and viral suppression [31].

Although antenatal viral load was not taken into consideration for the mode of delivery in this study, the rate of mother to child transmission of HIV was very low $2.2 \%(4 / 179)$ as compared the $22 \%$ got from a survey in the same country [4], questioning the necessity of antenatal viral load assessment in option B+ not only in low resources settings as this study seems to show. The meantime to achieve an undetectable viral load when starting HAART during pregnancy can be 60 days (as short as 12 days to 168 days) [32], the time to viral suppression depends on the baseline viral load [33], and, in the University Teaching Center in Yaoundé one of the study facility, we suppressed the viral load of a pregnant woman diagnosed HIV+ at the $36^{\text {th }}$ week with 70,000 copies $/ \mathrm{ml}$ with the option $\mathrm{B}+\mathrm{ART}$ protocol used in this study within 21 days. Concerning the four cases of HIV+ children, we cannot exclude the possibility of in utero transmission before the beginning of HAART, since none of them was on pre-conceptional ART [34].

One of the advantages of the option $\mathrm{B}+$ in low resources environment is that it can be implemented in any health facility level. It only needs routine confirmatory HIV testing, antiretroviral drugs given by any medical personnel in a task shifting strategy [35]. Option A and B were indeed dependent on accessible, functional, and efficient laboratory services for CD4 count testing [36]. It is therefore an important tool for the elimination of vertical and horizontal transmission of HIV [14].

HAART reduces milk HIV-1 mRNA which was the most frequent HIV type in our study more than zidovudine/nevirapine [37]. Even the three mothers who used the non-recommended mixed feeding had HIV free newborns.

The issue of obstetrical, early and long term postnatal complications due to fetal exposure to HAART is still debated. In India, it has been shown that women on HAART without protease inhibitors as it's the case in option B+ increased the risk of adverse pregnancy outcomes like preterm births and low birth weight children compared to AZT regimen [38]. In Cameroon, HAART initiated during pregnancy (option $\mathrm{B}+$ ) was also related to low birth weight [39]. Our low birth weight and prematurity rate were low but our study design couldn't allow us to conclude.

\section{Conclusion}

HIV during pregnancy concerned pregnant women of all ages, blood donation and ANC HIV screening remained the main HIV screening opportunity, self-decided spontaneous HIV screening behavior was rare and yet to be a routine, but once screened positive, the ART compliance was very good. The HIV status of the male partner was unknown. The mother to child transmission of HIV was very low and was not related to the number of antenatal visits, gesta- 
tional age of delivery, mode of delivery, birth weight or post-natal feeding. Antenatal viral load assessment in case of HAART taken for at least 4 weeks or elective caesarian section in a context of option $\mathrm{B}+$ are probably outdated strategies for PMTCT of HIV.

\section{Conflicts of Interest}

This work was not sponsored by any organization and the authors declare no conflict of interest.

\section{References}

[1] Global Aids Update (2017) Ending AIDS Progress towards the 90-90-90-Targets. http://www.unaids.org/sites/default/files/media_asset/Global_AIDS_update_2017_e $\underline{\text { n.pdf }}$

[2] UNAIDS (2016) Children and HIV in Fact Sheet. UNAIDS. http://www.unaids.org/sites/default/files/media_asset/FactSheet_Children_en.pdf

[3] Billong, S.-C., Fokam, J., Billong, E.-J., Nguefack-Tsague, G., Essi, M.J., Fodjo, R., et al. (2015) Epidemiological Distribution of HIV Infection among Pregnant Women in the Ten Regions of Cameroon and Strategic Implications for Prevention Programs. Pan African Medical Journal, 20, 79-87.

[4] Nguefack, H., Gwet, H., Desmonde, S., Oukem-Boyer, O., Nkenfou, C., Tejiokem, M., et al. (2016) Estimating Mother-to-Child HIV Transmission Rates in Cameroon in 2011: A Computer Simulation Approach. BMC Infectious Diseases, 16, 11-21. https://doi.org/10.1186/s12879-016-1336-2

[5] Adetokunboh, O.O. and Oluwasanu, M. (2016) Eliminating Mother-to-Child Transmission of the Human Immunodeficiency Virus in Sub-Saharan Africa: The Journey So Far and What Remains to Be Done. Journal of Infection and Public Health, 9, 396-307. https://doi.org/10.1016/j.jiph.2015.06.010

[6] Shapiro, R.L. and Lockman, S. (2010) Mortality among HIV-Exposed Infants: The First and Final Frontier. Clinical Infectious Diseases, 50, 445-447. https://doi.org/10.1086/649887

[7] Moges, N.A., Kassa, G.M. and Boneya, D.J. (2017) Rate of HIV Transmission and Associated Factors among HIV-Exposed Infants in Selected Health Facilities of East and West Gojjam Zones, Northwest Ethiopia; Retrospective Cohort Study. BMC Infectious Diseases, 17, 475.

https://www.ncbi.nlm.nih.gov/pmc/articles/PMC5501065/pdf/12879_2017_Article_ 2578.pdf

[8] Clayden, P. (2017) Option B+ Malawi. HIV Treatment Bulletin. http://i-base.info/htb/31787

[9] Guay, L.A., Musoke, P., Fleming, T., Bagenda, D., Allen, M., Nakabiito, C., Sherman, J., et al. (1999) Intrapartum and Neonatal Single-Dose Nevirapine Compared with Zidovudine for Prevention of Mother-to-Child Transmission of HIV-1 in Kampala, Uganda: HIVNET 012 Randomized Trial. Lancet, 354, 795-802. https://doi.org/10.1016/S0140-6736(99)80008-7

[10] World Health Organization (WHO) (2012) Use of Antiretroviral Drugs for Treating Pregnant Women and Preventing HIV Infection in Infants. WHO, Geneva. http://apps.who.int/iris/bitstream/handle/10665/70892/WHO_HIV_2012.6_eng.pdf ;jsessionid $=809 \mathrm{D} 3 \mathrm{D} 5 \mathrm{D} 3 \mathrm{CD} 096 \mathrm{D} 347166 \mathrm{BE} 55 \mathrm{BDE} 4 \mathrm{E} 8 \mathrm{E} ?$ sequence $=2$ 
[11] de Ruiter, A., Taylor, G.P., Clayden, P., Dhar, J., Gandhi, K., Gilleece, Y., et al. (2014) British HIV Association Guidelines for the Management of HIV Infection in Pregnant Women 2012 (2014 Interim Review). HIV Medicine, 15, 1-77. https://doi.org/10.1111/hiv.12185

[12] UNAIDS (2016) Global AIDS Update. http://www.unaids.org/sites/default/files/media_asset/global-AIDS-update-2016_en .pdf

[13] Wonga, V.J., Murray, K.R., Phelpsa, B.R., Vermund, S.H. and McCarraher, D.R. (2017) Adolescents, Young People, and the 90-90-90 Goals: A Call to Improve HIV Testing and Linkage to Treatment. AIDS, 31, S191-S194. https://doi.org/10.1097/QAD.0000000000001539

[14] Cohen, M.S., Chen, Y.Q., McCauley, M., Gamble, T., Hosseinipour, M.C., Kumarasamy, N., et al. (2011) Prevention of HIV-1 Infection with Early Antiretroviral Therapy. The New England Journal of Medicine, 365, 493-505. https://doi.org/10.1056/NEJMoa1105243

[15] Cameroun (2015) Multiple Indicator Cluster Survey (MICS) 2014 (Enquête par grappe à indicateurs multiples 2014. Rapport de résultats clés.

https://mics-surveys-prod.s3.amazonaws.com/MICS5/West\%20and\%20Central\%20 Africa/Cameroon/2014/Key\%20findings/Cameroon\%202014\%20MICS\%20KFR_French .pdf

[16] Nannozi, V., Wobudeya, E. and Gahagan, J. (2016) Fear of an HIV Positive Test Result: An Exploration of the Low Uptake of Couples HIV Counseling and Testing (CHCT) in a Rural Setting in Mukono District, Uganda. Global Health Promotion, 24, 33-42.

[17] Wolf, H.T., Halpern-Felsher, B.L., Bukusi, E.A., Agot, K.E., Cohen, C.R. and Auerswald, C.L. (2014) It Is All about the Fear of Being Discriminated [against] ... the Person Suffering from HIV Will Not Be Accepted: A Qualitative Study Exploring the Reasons for Loss to Follow-Up among HIV-Positive Youth in Kisumu, Kenya. BMC Public Health, 14, 1154. https://doi.org/10.1186/1471-2458-14-1154

[18] Qiao, S., Zhang, Y., Li, X. and Menon, J.A. (2018) Facilitators and Barriers for HIV-Testing in Zambia: A Systematic Review of Multi-Level Factors. PLoS ONE, 13, e0192327. https://doi.org/10.1371/journal.pone.0192327

[19] Nöstlinger, C., Rojas Castro, D., Platteau, T., Dias, S. and Le Gall, J. (2014) HIV-Related Discrimination in European Health Care Settings. AIDS Patient Care and STDs, 28, 155-161. https://doi.org/10.1089/apc.2013.0247

[20] WHO and UNAIDS (2010) Addressing Violence against Women and HIV/AIDS: What Works?

http://apps.who.int/iris/bitstream/handle/10665/44378/9789241599863_eng.pdf;jses sionid=09202B82DCA11802635F793DB1D0DAB9? sequence $=1$

[21] Branson, B.M., Handsfield, H.H., Lampe, M.A., Janssen, R.S., Taylor, A.W., Lyss, S.B., et al. (2006) Centers for Disease Control and Prevention (CDC). Revised Recommendations for HIV Testing of Adults, Adolescents, and Pregnant Women in Health-Care Settings. MMWR Recommendations and Reports, 55, 1-17. https://www.cdc.gov/mmwr/preview/mmwrhtml/rr5514a1.htm

[22] Henriquez-Camacho, C., Villafuerte-Gutierrez, P., Pérez-Molina, J.A., Losa, J., Gotuzzo, E. and Cheyne, N. (2017) Opt-Out Screening Strategy for HIV Infection among Patients Attending Emergency Departments: Systematic Review and Me- 
ta-Analysis. HIV Medicine, 18, 419-429. https://doi.org/10.1111/hiv.12474

[23] Ngo Nonga, B., Billong, S.C., Thek, P., Mve, V., Tiyou, C., Ambassa, B., et al. (2016) Factors That May Influence Adherence in a University-Based Program for the Prevention of Mother-to-Child Transmission of HIV in Yaoundé-Cameroon. IJTDH, 14, 1-6.

[24] Dionne-Odom, J., Thomas, K., Welty, T.K., Westfall, A.O., Chi, B.H., Koumavi Ekouevi, D., Kasaro, M., Tih, P.M. and Tita, A.T.N. (2016) Factors Associated with PMTCT Cascade Completion in Four African Countries. AIDS Research and Treatment, 2016, Article ID: 2403936.

[25] Gugsa, S., Potter, K., Tweya, H., Phiri, S., Sande, O., Sikwese, P., et al. (2017) Exploring Factors Associated with ART Adherence and Retention in Care under Option B+ Strategy in Malawi: A Qualitative Study. PLoS ONE, 12, e0179838. https://doi.org/10.1371/journal.pone.0179838

[26] Dalal, S., Johnson, C., Fonner, V., Kennedy, C.E., Siegfried, N., Figueroa, C., et al. (2017) Improving HIV Test Uptake and Case Finding with Assisted Partner Notification Services. AIDS, 31, 1867-1876. https://doi.org/10.1097/QAD.0000000000001555

[27] Flax, V.L., Yourkavitch, J., Okello, E.S., Kadzandira, J., Katahoire, A.R. and Munthali, A.C. (2017) If My Husband Leaves Me, I Will Go Home and Suffer, So Better Cling to Him and Hide This Thing: The Influence of Gender on Option B+ Prevention of Mother-to-Child Transmission Participation in Malawi and Uganda. PLoS ONE, 12, e0178298. https://doi.org/10.1371/journal.pone.0178298

[28] (2008) Malawi, HIV and AIDS. Monitoring and Evaluation Report 2007-2008. National AIDS Commission, Lilongwe.

https://www.google.cm/search?dcr=0\&ei=CeN-W-GJAo_cwQKr9pfQBw\&q=Mala wi+hiv+and+aids+monitoring+and+evaluation+report+2008\&oq=Malawi+hiv+an $\mathrm{d}+$ aids+monitoring+and+evaluation+report+2008\&gs_l=psy-ab.12...455672.499349 $.0 .502607 .87 .35 .0 .29 .29 .0 .2265 .5252 .5-2 \mathrm{j} 2 \mathrm{j} 9-1.5 .0 \ldots . .0 \ldots 1 \mathrm{c} .1 .64 . \mathrm{psy}-\mathrm{ab} . .53 .33 .5023 \ldots 0 \mathrm{i}$ 19k1j33i22i29i30k1j0i22i30k1.0.xDwi5ZGDSts

[29] Caliari, J.S., Teles, S.A., Reis, R.K. and Gir, E. (2017) Factors Related to the Perceived Stigmatization of People Living with HIV. Revista da Escola de Enfermagem da USP, 51, e03248. https://doi.org/10.1590/s1980-220x2016046703248

[30] Read, J.S. and Newell, M.K. (2005) Efficacy and Safety of Cesarean Delivery for Prevention of Mother-to-Child Transmission of HIV-1. Cochrane Database of Systematic Reviews, 19, CD005479.

https://www.ncbi.nlm.nih.gov/pubmed/16235405

[31] Kennedy, C.E., Yeh, P.T., Pandey, S., Betran, A.P. and Narasimhan, M. (2017) Elective Cesarean Section for Women Living with HIV: A Systematic Review of Risks and Benefits. AIDS, 31, 1579-1591. https://doi.org/10.1097/QAD.0000000000001535

[32] Snippenburg van, W., Nellen, F.J.B., Smit, C., Wensing, A.M.J., Godfried, M.H. and Mudrikova, T. (2017) Factors Associated with Time to Achieve an Undetectable HIV RNA Viral Load after Start of Antiretroviral Treatment in HIV-1-Infected Pregnant Women. Journal of Virus Eradication, 3, 34-39.

[33] Read, P.J., Mandalia, S., Khan, P., Harrisson, U., Naftalin, C., Gilleece, Y., et al. (2012) When Should HAART Be Initiated in Pregnancy to Achieve an Undetectable HIV Viral Load by Delivery? AIDS, 26, 1095-1103. https://doi.org/10.1097/QAD.0b013e3283536a6c

[34] Hoffman, R.M., Black, V., Technau, K., van der Merwe, K.J., Currier, J., Coovadia, 
A., et al. (2010) Effects of Highly Active Antiretroviral Therapy Duration and Regimen on Risk for Mother-to-Child Transmission of HIV in Johannesburg, South Africa. JAIDS Journal of Acquired Immune Deficiency Syndromes, 54, 35-41. https://doi.org/10.1097/QAI.0b013e3181cf9979

[35] Kalua, T., Tippett Barr, B.A., van Oosterhout, J.J., Mbori-Ngacha, D., Schouten, E.J., Gutpa, S., et al. (2017) Lessons Learned from Option b+ in the Evolution toward "Test and Start" from Malawi, Cameroon, and the United Republic of Tanzania. JAIDS Journal of Acquired Immune Deficiency Syndromes, 75, S43-S50. https://doi.org/10.1097/QAI.0000000000001326

[36] Shouten, E., Jahn, A., Chimbwandira, F., Harries, A.D. and Van Damme, W. (2013) Is Option B+ the Best Choice. The Lancet, 381, 1272-1273. https://doi.org/10.1016/S0140-6736(13)60833-8

[37] Chung, M.H., Kiarie, J.N. and Barbra, A. (2008) Highly Active Antiretroviral Therapy (HAART) versus Zidovudine/Nevirapine Effects on Early Breast Milk HIV-1 RNA: A Phase II Randomized Clinical Trial. Antiviral Therapy, 13, 799-807.

[38] Darak, S., Darak, T., Kulkarni, S., Kulkarni, V., Parchure, R., Hutter, I., et al. (2013) Effect of Highly Active Antiretroviral Treatment (HAART) during Pregnancy on Pregnancy Outcomes: Experiences from a PMTCT Program in Western India. AIDS Patient Care STDS, 27, 163-170. https://doi.org/10.1089/apc.2012.0401

[39] Njom Nlend, A.E., Nga Motazé, A., Moyo Tetang, S., Zeudja, C., Ngantcha, M. and Tejiokem, M. (2016) Preterm Birth and Low Birth Weight after in Utero Exposure to Antiretrovirals Initiated during Pregnancy in Yaoundé, Cameroon. PLoS ONE, 11, e0150565. https://doi.org/10.1371/journal.pone.0150565 